www.nature.com/jhg

\title{
EDITORIAL
}

\section{New Year's Greetings}

Journal of Human Genetics (2012) 57, 1-2; doi:10.1038/jhg.2011.141

$\mathrm{W}$ elcome to the first 2012 issue of the Journal of Human Genetics (JHG).

It would be obvious to most readers that in recent times, we have been publishing a greater number of papers submitted from countries other than Japan. More importantly, more papers are being submitted from regions relatively new to research in the field of human genetics and, encouragingly, these are also being published in JHG. I really welcome this trend. I think this is also a good reflection of the journal's improved visibility, especially on the internet, which is proved by the $40 \%$ increase of regular visitors to the journal's site compared with 3 years ago, before JHG's new era started with Nature Publishing Group. JHG is growing.

In 2012, to ensure that the journal continues to grow, I would like to start thinking about new strategies that will take JHG onto the 'next stage'. JHG still has a lot of room for further improvement and growth to be a truly international premier journal of human genetics whilst remaining an official journal of the Japan Society of Human Genetics. We need to consider ways to successfully achieve our future goals and also attract a greater number of high-quality submissions. To further improve the efficiency in the editorial and peer-reviewing processes, to review the journal's scope and define the journal's goals, with a

priority for future growth, are the important tasks for us in this coming year. Through active discussion among the editors and parent society members, I would like to develop the best methods to accomplish these tasks. Of course, I would also be happy to receive ideas and feedback from our readers and authors.

I thank all of our authors, readers and reviewers for their kind and continued participation.

Here's to another busy year in 2012. A very happy New Year!

\section{ACKNOWLEDGEMENTS}

The Editor-in-Chief, on behalf of the JHG Editorial Board and the Japan Society of Human Genetics, gratefully acknowledges the generous support from peer reviewers in carefully assessing manuscripts under consideration. The following individuals provided reviews of papers submitted to JHG in 2011:

Katsushi Tokunaga Editor-in-Chief Journal of Human Genetics

Fuchang, Zhang

Fujimoto, Akihiro

Fujita, Tomoe

Fukumaki, Yasuyuki

Funayama, Manabu

Furukawa, Yoichi

Galfalvy, Hanga

Gojobori, Jun

Goloni-Bertollo, Eny Maria

Goto, Yu-Ichi

Guan, Minxin

Haga, Nobuhiko

Hamada, Hiromichi

Harada, Naoki

Hasegawa, Tomonobu

Hashimoto, Ryota

Hata, Ken-ichirou

Hayasaka, Kiyoshi

Hayashi, Shin

Hayashi, Yukiko

Heyer, Evelyne

Hildebrand, Michael S

Hirasawa, Akira
Hiratsuka, Masahiko

Hishimoto, Akitoyo

Hiyama, Toru

Hofreiter, Michael

Horikawa, Yukio

Hosoi, Takayuki

Hotta, Kikuko

Ihara, Kenji

Iijima, Kazumoto

Ikeda, Akio

Ikegawa, Shiro

Ikezono, Tetsuo

Imoto, Issei

Inoue, Ituro

Inoue, Jun

Ishikawa, Kinya

Ishikawa, Shumpei

Ishioka, Chikashi

Isomura, Minoru

Iwamoto, Sadahiko

Iwasaki, Naoko

Iwasaki, Satoshi

Jezewski, Peter
Bunyavanich, Supinda

Burger, Joachim
Filippo, Cesare

Fogli, Anne 
Johnson, Todd Andrew Jongjaroenprasert, Wallaya

Kamatani, Naoyuki

Kamatani, Yoichiro

Kamide, Kei

Kaname, Tadashi

Karasugi, Tatsuki

Katagiri, Toyomasa

Kato, Mitsuhiro

Kato, Tadafumi

Katsuya, Tomohiro

Katsuyama, Hironobu

Kawakami, Kiyoshi

Kawame, Hiroshi

Keicho, Naoto

Kidd, Kenneth K

Kido, Hiroshi

Kimura, Akinori

Kishino, Tatsuya

Kitajiri, Shin-ichiro

Knijff, Peter

Kobashi, Gen

Kobayashi, Kazuhiro

Kochi, Yuta

Kohara, Katsuhiko

Koizumi, Akio

Kong, Qing-Ping

Kosaki, Kenjiro

Koshimizu, T

Kosho, Tomoki

Kotzot, Dieter

Koyama, Satomi

Kubota, Takeo

Kumasaka, Natsuhiko

Kurahashi, Hiroki

Kure, Shigeo

Kurosawa, Kenji

Kurosawa, Motohiro

Lao, Oscar

Lau, Yu-Lung

Lee, Ming Ta Michael

Lewis, Cathryn

Li, Hui

Li, Ling-Hui

Li, Shengbin

Li, Wei

Lin, Dongxin

Liu, Xue Zhong

Ma, Qing

Maeda, Jun

Maeda, Shiro

Mahasirimongkol, Surakameth

Mahdieh, Nejat

Makita, Yoshio

Malhi, Ripan S

Malyarchuk, Boris A

Mann, Kathy

Mano, Shuhei
Marioni, RE

Masuda, Satohiro

Masumoto, Hiroshi

Mathur, Premendu Prakash

Matsubara, Yoichi

Matsuda, Koichi

Matsunaga, Tatsuo

Matsushita, Kazuyuki

McElreavey, Ken

Miki, Takashi

Minerva, Carrasquillo

Misawa, Kazuharu

Miura, Masatomo

Miyata, Satoshi

Miyazaki, Tatsuhiko

Mizuno, Toshiki

Mizuta, Ikuko

Morisaki, Hiroko

Morishita, Shinnichi

Mundlos, Stefan

Murakami, Yoshinori

Muramatsu, Masaaki

Mushiroda, Taisei

Mutirangura, Apiwat

Muto, Masahiko

Muzzio, Marina

Nabika, Toru

Nakagawa, Hidewaki

Nakagawa, Masanori

Nakagome, Shigeki

Nakajima, Atsushi

Nakajima, Toshiaki

Nakamura, Kimitoshi

Nakano, Yoshiro

Nakazawa, Mitsuru

Nawa, Hiroyuki

Nemer, Georges

Niikawa, Norio

Nikawa, Ken

Nishimura, Gen

Nishino, Ichizo

Noguchi, Emiko

Nothnagel, Michael

Nuchnoi, Pornlada

Ogimoto, Akiyoshi

Oh, Bermseok

Ohashi, Hirofumi

Ohashi, Jun

Ohira, Miki

Ohta, Tohru

Okada, Yukinori

Okamoto, Nobuhiko

Olivieri, Anna

Onodera, Osamu

Onouchi, Yoshihiro

Onuma, Hiroshi

Oppenheimer, Stephen

Osawa, Haruhiko
Oven, Mannis van

Ozaki, Kouichi

Ozawa, Nobuaki

Paisan-Ruiz, Coro

Parisiadou, Loukia

Park, Ji Wan

Pereira, Luisa

Perera, M

Poloni, Estella S

Rao, Lakshmi

Ray, Kunal

Reuser, Arnold

Rickards, Olga

Sáenz, Amets

Saitoh, Shinji

Saitou, Naruya

Saitsu, Hirotomo

Sakai, Norio

Sakamoto, Osamu

Sakurai, Akihiro

Salas, Antonio

Sasaki, Hidenao

Satake, Wataru

Sato, Shinichi

Sediva, Anna

Seeman, Pavel

Seki, Naohiko

Sengupta, Sanghamitra

Sharma, Swarkar

Shaw, Philip

Shi, Hong

Shimizu, Norikazu

Shimozawa, Nobuyuki

Shinozawa, Keiko

Shintaku, Haruo

Sinha, Pratima

Song, Kyuyoung

Sorimachi, Hiroyuki

Stanislawska-Sachadyn, Anna

Steinlein, Ortrud K

$\mathrm{Su}$, Bing

Suchanek, Renata

Sueoka, Kou

Sugano, Kokichi

Sugie, Hideo

Suktitipat, Bhoom

Sun, Chang

Sun, Xueying

Suriyapol, Prapat

Suzuki, Akari

Suzuki, Tamio

Suzuki, Yasuyuki

Suzuki, Yoichi

Tabara, Yasuharu

Tajima, Atsushi

Tajima, Go

Takeshita, Haruo

Takeuchi, Fumihiko
Tamari, Mayumi

Tamiya, Gen

Tamura, Kazuo

Tanaka, Toshihiro

Tang, Nelson

Tang, Tiong Yang

Taniguchi, Yoshihito

Tashiro, Kei

Taylor, Robert W

Thangaraj, Kumarasamy

Thiele, Susanne

Tim, Chew Fook

Tomatsu, Shunji

Tomita, Hiroaki

Trejaut, Jean Alain

Tsuchiya, Naoyuki

Tsujikawa, Motokazu

Tsukaguchi, Yasuhiro

Tsunoda, Tatsuhiko

Usami, Shin-ichi

Vatta, Matteo

Villard, Laurent

Vona, Giuseppe

Wada, Takahito

Wadelius, M

Wang, Wei

Watanabe, Atsushi

Wei, Qingyi

Wiklund, Fredrik

Wilson, James $\mathrm{F}$

Wolf, Herman Maximilian

$\mathrm{Wu}$, Bailin

Wu, Ying

$\mathrm{Xu}$, Shuhua

Yamada, Ryo

Yamada, Yoshiji

Yamaguchi-Kabata, Yumi

Yamakawa, Kazuhiro

Yamakawa-Kobayashi, Kimiko

Yamamoto, Ken

Yamamoto, Koujirou

Yamamoto, Toshiyuki

Yang, Zhenglin

Yao, Yong-Gang

Yasuda, Kazuki

Yen, Pauline

Yin, G

Yin, Jiaoyang

Yokoi, Sana

Yokoi, Tsuyoshi

Yoshida, Kiyotsugu

Yoshida, Kunihiro

Yoshida, Masaru

Yoshikawa, Takeo

Yoshimura, Kunihiko

Yoshiura, Koh-ichiro

Zappavigna, Vincenzo

Zhou, Gangqiao 